\title{
Desnervação capsular percutânea ou aberta no tratamento da dor na displasia coxofemoral canina
}

\author{
Percutaneous versus open hip denervation in the treatment of canine pain hip dysplasia
}

\author{
Gisele Francine da Silva' Amanda Viana Carvalho ${ }^{\text {II }}$ Bianca Mota Penteado ${ }^{\text {III }}$ \\ Bruno Testoni Lins ${ }^{\mathrm{IV}}$ André Luis Selmi ${ }^{\mathrm{III}, \mathrm{IV}^{*}}$
}

\section{RESUMO}

A desnervação capsular (DC) se baseia na desperiostização da borda acetabular e tem sido utilizada como uma das técnicas de alívio da dor articular em cães displásicos. Compararam-se as técnicas de DC percutânea e aberta, aplicadas em 25 cães, que apresentaram diagnóstico clínico e radiográfico de displasia coxofemoral, totalizando 31 cirurgias. Os efeitos das técnicas foram comparados, em período de 180 dias, por meio de avaliação subjetiva de claudicação e dor, perimetria da coxa, graus máximos de flexão e extensão da articulação coxofemoral e amplitude de movimento articular. Os graus de claudicação e dor apresentaram decréscimo significativo ao longo do período avaliado em ambos os grupos, sem apresentarem diferenças quando comparados entre si. Com relação à perimetria da coxa, flexão, extensão e amplitude de movimento articular, foi observado o aumento dos valores numéricos destas variáveis ao longo do estudo. Baseado nos achados, concluiu-se que a abordagem percutânea ou aberta na DC produz similitude nas variáveis avaliadas, e ambas trazem bons resultados.

Palavras-chave: doença articular degenerativa, incongruência articular, claudicação, cães.

\section{ABSTRACT}

Joint capsule denervation is based on removal of sensitive fibers from the acetabular edge and has been used as one of the techniques to joint pain relief in the canine dysplastic hip. This study compared the techniques of percutaneous and open hip denervation in 25 dogs that showed clinical and radiographic signs of hip dysplasia, totaling 31 surgeries. The effects of the techniques were evaluated and compared in a period of 180 days following surgery, including subjective evaluation of lameness and pain, thigh girth, maximum degree of hip flexion and extension and range of motion (ROM). Degree of lameness and pain significantly decreased during the study period for both groups, and no difference could be observed between groups. Thigh girth, degree of flexion, extension and ROM presented an increase in the numerical values throughout the study. Based on these findings, it is concluded that the percutaneous or open approach for capsular denervation produced similar results.

Key words: degenerative joint disease, articular incongruence, lameness, dogs.

\section{INTRODUÇÃO}

A displasia coxofemoral (DCF) nos cães pode ser definida como uma afecção biomecânica representada pela disparidade entre massa muscular primária e o rápido crescimento ósseo, que pode levar a doença articular degenerativa (DAD) de forma progressiva e dolorosa, especialmente em cães de raças de grande porte e gigantes (HULSE \& JOHNSON, 2002; RAWSON et al., 2005).

As causas são multifatoriais, incluindo fatores hereditários, ambientais, nutricionais e hormonais, entre outros (ARNBJERG, 1999; HULSE \& JOHNSON, 2002). O histórico e sinais clínicos usualmente incluem anormalidades no andar, tal como

IUniversidade Anhembi Morumbi (UAM), São Paulo, SP, Brasil.

IIPrograma de Pós-graduação, UAM, São Paulo, SP, Brasil.

IIIPrograma de Mestrado em Cirurgia e Anestesiologia Veterinária, Universidade de Franca (UNIFRAN), Franca, SP, Brasil.

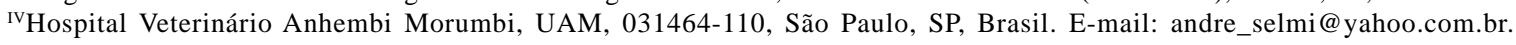

*Autor para correspondência. 
claudicação, passos curtos, diminuição na tolerância ao exercício e dificuldade em levantar-se e subir degraus (TOMLINSON \& MCLAUGHLIN, 1996; HULSE \& JOHNSON, 2002; RAWSON et al., 2005; SELMI et al., 2009).

O diagnóstico da DCF é baseado na história relatada pelo proprietário, nos sinais clínicos e nos resultados específicos do exame ortopédico da articulação coxofemoral. O diagnóstico definitivo é fornecido pela avaliação radiográfica, evidenciando flacidez da cápsula articular, deformações osteoarticulares e presença de osteoartrose. A intensidade das alterações radiográficas difere daquela relacionada aos sinais clínicos (SCHULZ, 2008). O prognóstico cirúrgico está relacionado à gravidade da displasia, assim como o grau de atrofia muscular, sendo que, quanto maior a atrofia muscular no membro atingido, maior o tempo de convalescença até se atingir a melhora (BRAUN et al., 2003).

Diversos tratamentos têm sido utilizados para controle, correção ou alívio desta afecção, sendo as modalidades conservativa e cirúrgica descritas. A desnervação capsular (DC) está entre as técnicas cirúrgicas que promovem alívio da dor e baseia-se na desperiostização da borda acetabular cranial e dorsal (KINZEL et al., 1998; SCHAMAEDECKE, 2004), podendo ser por abordagem percutânea ou aberta (SELMI et al., 2009).

Poucos estudos têm abordado os resultados da DC em cães e, recentemente, FERRIGNO et al. (2007b), SELMI et al. (2009) e LISTER et al. (2009) descreveram os resultados da técnica, por meio de avaliações subjetivas e objetivas. O objetivo deste estudo foi comparar os resultados clínicos da técnica de DC por meio das abordagens percutânea e aberta, em cães adultos, com sinais clínicos e radiográficos de DCF.

\section{MATERIAL E MÉTODOS}

A técnica de DC percutânea e DC aberta foi aplicada em 25 cães com diagnóstico clínico e radiográfico de DCF, uni ou bilateral, sintomáticos, apresentando ou não DAD, totalizando 31 cirurgias. Não foram realizadas limitações referentes a gênero, raça, peso ou idade para inclusão neste estudo.

Os cães foram alocados em dois grupos, sendo o grupo um (GI) submetido à técnica de DC percutânea $(n=14)$, totalizando 14 cirurgias, e o grupo dois (GII) submetido à DC aberta $(\mathrm{n}=11)$, sendo seis cães bilateralmente, porém não simultâneas, totalizando 17 cirurgias. $\mathrm{O}$ intervalo entre as cirurgias bilaterais foi de 19 \pm 2.69 . Para fins de aferição, cada procedimento cirúrgico unilateral determinou o momento inicial de avaliação de cada um dos lados operados. Quatro cães foram desconsiderados de GII, pois, em dois deles, não foi possível realizar as mensurações em todos os tempos pré-determinados e os outros dois receberam medicação anti-inflamatória nos três dias que antecederam à intervenção cirúrgica. A escolha dos cães para cada grupo foi realizada de forma aleatória por meio de sorteio do tipo "cara” ou "coroa”.

Os graus de claudicação e dor foram avaliados por escala analógica visual, segundo recomendações de HUDSON et al. (2004), realizado pelo proprietário e pelo autor deste estudo, de forma independente. O perímetro muscular da coxa foi mensurado imediatamente distal à prega inguinal por meio de fita métrica inelástica; os ângulos máximos de flexão e extensão passivos da articulação do quadril foram mensurados por meio de goniômetro plástico com o centro de rotação posicionado sobre o trocânter maior do fêmur. A amplitude de movimento articular (APM) foi adquirida através dos valores da extensão e flexão passivos da articulação. Cada mensuração objetiva foi realizada em triplicata pelo mesmo avaliador.

Todas as mensurações foram realizadas no momento prévio à cirurgia e após sete, 15, 30 e 180 dias de pós-operatório. Para tais aferições, os animais não foram sedados e não estavam recebendo medicações anti-inflamatórias por pelo menos três dias antes da cirurgia.

Os animais foram submetidos a exames de rotina pré-operatórios, além de outros julgados necessários na avaliação individual de cada caso. Para a intervenção cirúrgica, cada cão passou pelos procedimentos pré-cirúrgicos e anestésicos condizentes com o seu estado geral. No momento anterior ao procedimento cirúrgico, o paciente foi posicionado em decúbito lateral e a articulação a ser operada posicionada dorsalmente.

No GI, a abordagem foi realizada de acordo com SELMI et al. (2009), localizando-se aproximadamente um centímetro cranial e dorsal a porção mais proximal do trocânter maior, seguida de pequena incisão cutânea com lâmina de bisturi $n^{0} \mathbf{1 5}$, pela qual se introduziu, a campo fechado, pino intramedular com ponta trifacetada de 3,5-mm de diâmetro, até atingir o corpo do ílio. Posteriormente, foram realizados movimentos repetidos de raspagem do periósteo, em sentido semicircular, no aspecto cranial, crânio-dorsal e dorsal da borda acetabular, tomando-se cuidado para evitar a perda de contato da ponta do pino intramedular com a superfície óssea no aspecto dorsolateral e ventrolateral do corpo ilíaco. A ferida cutânea foi cerrada por meio de fio de sutura inabsorvível náilon 2-0, em padrão interrompido simples. 
No GII, a abordagem para a realização da desnervação foi realizada pelo método modificado de FERRIGNO et al. (2004), por meio de incisão cutânea no eixo formado entre o trocânter maior e o corpo do ílio, com aproximadamente cinco centímetros de extensão. A seguir, foi efetuada a divulsão dos tecidos subjacentes, a localização da fáscia lata entre o músculo glúteo médio e tensor da fáscia lata. O músculo glúteo médio foi rebatido dorsalmente e o glúteo profundo ventralmente. Realizou-se curetagem para remoção do periósteo da margem acetabular cranio-dorsal e dorsal, utilizando-se curetas de Volkmann. A região foi lavada com solução fisiológica, seguindo-se com a aproximação da fáscia entre o músculo glúteo médio e tensor da fáscia lata e do tecido subcutâneo, por meio de fio de poliglecaprone 2-0, em padrão simples contínuo. A pele foi ocluída com náilon 2-0, com padrão colchoeiro horizontal contínuo.

No pós-operatório, para a uniformidade das avaliações, os animais de ambos os grupos receberam o mesmo protocolo medicamentoso: anti-inflamatório nãoesteroidal a base de carprofeno, na dose de 2,2-mg kg-1, a cada 12 horas, durante três dias, antibióticos a base de enrofloxacina, na dose única diária de 5,0-mg kg-1 , durante sete dias, dipirona, na dose de $25,0 \mathrm{mg} \mathrm{kg}^{-1}$, a cada oito horas, durante cinco dias e cloridrato de ranitidina na dose de 2,0mg kg-1 a cada 12 horas, por sete dias.

Os dados paramétricos e não paramétricos foram analisados pelo método estatístico de ANOVA, seguido pelo teste de Bonferroni para avaliar o efeito do procedimento ao longo do período de avaliação dentro de cada grupo e de cada momento entre os grupos. Adotou-se grau de significância de $5 \%(P<0,05)$.

\section{RESULTADOS E DISCUSSÃO}

Todos os cães inclusos para avaliação neste estudo (10 machos e 11 fêmeas, perfazendo 27 intervenções cirúrgicas) eram adultos e apresentaram sinais clínicos compatíveis, com dor na articulação coxofemoral e tiveram, ao exame radiográfico, diagnóstico de DCF. A média de idade e peso dos cães em GI foi de 4,78 $\pm 1,88$ anos e $29,42 \pm 6,08 \mathrm{~kg}$, e GII $7,57 \pm 2,14$ anos e $36,21 \pm 6,76 \mathrm{~kg}$, respectivamente, não houve diferença significativa entre os grupos.

Este estudo demonstrou a eficácia da DC pelas abordagens aberta e percutânea, após 180 dias de avaliação em $90 \%$ dos animais, já que ambas as abordagens demonstraram diminuição significativa nas variáveis de claudicação e dor. SELMI et al. (2009), utilizando a técnica de DC percutânea, descreveram melhora significativa nos escores de claudicação e dor após 15 dias de avaliação, e estes se mantiveram estáveis em 95,7\% dos animais até 180 dias de pósoperatório. BRAUN et al. (2003), ao aplicar a DC aberta, relataram a satisfação do proprietário em 93\% de 43 cães operados. Da mesma forma, KINZEL et al. (2002b), utilizando a mesma técnica em 295 cães, obtiveram melhora clínica em 92\% dos cães.

Todos os cães mantiveram o quadro estável, sem claudicação, ao longo do período avaliado (Tabela 1). Não foram observadas diferenças entre grupos quando estes foram comparados.

De acordo com os resultados apresentados para dor, com distribuição similar àqueles da claudicação, a diferença numérica dos valores entre GI e GII pode estar relacionada à dor que fora referida pelos proprietários do GI nos três primeiros dias de pós-operatórios, porém sem interferência estatística entre os grupos. Aos 180 dias de pós-operatório, o sucesso de pós-operatórios do GII foi de $84 \%$, enquanto o do GI permaneceu em 100\%; os animais apresentavam melhora significativa nos quadros de dor e claudicação.

SELMI et al. (2009), após empregarem a DC percutânea em 92 cães, sugeriram que os animais apresentaram menor trauma cirúrgico e menores riscos, principalmente pela evidente diminuição no tempo trans-operatório e pela baixa morbidade relacionada à diminuta incisão. No entanto, referiram à piora do quadro de dor em $67 \%$ dos pacientes nos três primeiros dias de pós-operatórios. Este trabalho corrobora os resultados apresentados naquele estudo, já que 57\% dos proprietários dos cães submetidos a mesma técnica referiram piora no quadro álgico nos três dias posteriores à intervenção cirúrgica.

De acordo com estudos anteriores, a dor pode estar relacionada à lesão muscular decorrente da perfuração e movimentação do pino intramedular durante a desnervação, a qual pode ocasionar neupraxia e lesão direta; tal achado apresentou resolução espontânea em até 14 dias em todos os animais do estudo. Para FERRIGNO et al. (2004), a DC aberta proporcionou melhora da dor já na primeira semana de pós-operatório em 91,4\% dos pacientes, especialmente após o 15ํㅡㄹ dia pós-operatório. Dados similares foram obtidos neste estudo, quando utilizada a DC aberta, com melhora clínica de 100\% dos cães com o mesmo período de pós-operatório.

Dois animais do GII apresentaram piora do quadro de dor e claudicação a partir dos 180 dias de pós-operatórios, o que também foi observado no estudo de ROCHA (2005), que relatou recidiva da dor em 10\% dos pacientes, e também por BRAUN et al. (2003), que observaram $32,6 \%$ de recidiva da dor em um período de 3,5 anos de avaliação, ambos utilizando a mesma técnica. LISTER et al. (2009) sugeriram que, 
Tabela 1 - Média e desvio-padrão dos escores de claudicação e dor atribuídos por meio de escala analógica visual; dos valores de perimetria da coxa, flexão e extensão máxima passiva da articulação coxofemoral e APM (amplitude de movimento articular) dos cães. Período de avaliação (0 a 180 dias)

\begin{tabular}{|c|c|c|c|c|c|}
\hline \multirow[t]{2}{*}{ Dor (escala de 0 a 10 ) } & \multicolumn{5}{|c|}{ 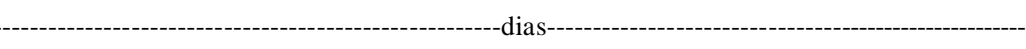 } \\
\hline & 0 & 7 & 15 & 30 & 180 \\
\hline Grupo I & $8,4 \pm 0,6^{*}$ & $1,1 \pm 0,4 *$ & $0,3 \pm 0,3^{*}$ & $0,0 \pm 0,0 *$ & $0,0 \pm 0,0^{*}$ \\
\hline Grupo II & $8,4 \pm 0,7 *$ & $4,8 \pm 2,8 *$ & $3,1 \pm 2,2 *$ & $1,6 \pm 2,0 *$ & $1,8 \pm 1,8 *$ \\
\hline \multirow[t]{2}{*}{ Claudicação (escala de 0 a 10) } & 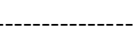 & $-x$ & -----dias-----. & ----------- & 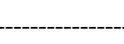 \\
\hline & 0 & 7 & 15 & 30 & 180 \\
\hline Grupo I & $8,2 \pm 0,1^{*}$ & $4,6 \pm 0,8^{*}$ & $1,2 \pm 0,3 *$ & $0,2 \pm 0,2^{*}$ & $0,0 \pm 0,0^{*}$ \\
\hline Grupo II & $7,5 \pm 1,6 *$ & $5,3 \pm 2,6 *$ & $3,1 \pm 1,7 *$ & $1,4 \pm 1,5^{*}$ & $1,4 \pm 1,4^{*}$ \\
\hline \multirow[t]{2}{*}{ Perimetria da coxa $(\mathrm{cm})$} & ------- & --------- & -----dias------ & 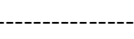 & - \\
\hline & 0 & 7 & 15 & 30 & 180 \\
\hline Grupo I & $36,4 \pm 4,3$ & $36,4 \pm 4,3$ & $36,5 \pm 4,3$ & $37,0 \pm 4,5$ & $37,9 \pm 4,8$ \\
\hline Grupo II & $40,3 \pm 2,4$ & $40,4 \pm 2,3$ & $41,1 \pm 2,5$ & $41,3 \pm 2,5$ & $42,5 \pm 2,2$ \\
\hline \multirow[t]{2}{*}{ Flexão máxima passiva $\left({ }^{\circ}\right)$} & - & & -----dias------ & ------- & 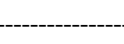 \\
\hline & 0 & 7 & 15 & 30 & 180 \\
\hline Grupo I & $48,6 \pm 2,5$ & $48,9 \pm 2,7$ & $48,5 \pm 2,3$ & $48,6 \pm 2,0$ & $48,5 \pm 1,7$ \\
\hline Grupo II & $59,3 \pm 5,4$ & $57,3 \pm 6,4$ & $54,9 \pm 5,3$ & $53,1 \pm 4,0$ & $53,7 \pm 4,9$ \\
\hline \multirow[t]{2}{*}{ Extensão máxima passiva $\left({ }^{\circ}\right)$} & -- & -------- & -----dias------ & --------------' & - \\
\hline & 0 & 7 & 15 & 30 & 180 \\
\hline Grupo I & $129,9 \pm 5,0$ & $129,5 \pm 4,8$ & $132,0 \pm 5,5$ & $138,8 \pm 7,4$ & $142,9 \pm 6,7$ \\
\hline Grupo II & $117,8 \pm 8,2$ & $126,2 \pm 8,4$ & $132,5 \pm 679$ & $136,4 \pm 6,7$ & $135,3 \pm 9,1$ \\
\hline \multirow[t]{2}{*}{ Amplitude de movimento articular $\left(^{\circ}\right)$} & & & ----dias------ & -1---- & -------- \\
\hline & 0 & 7 & 15 & 30 & 180 \\
\hline Grupo I & $81,2 \pm 2,5$ & $80,6 \pm 2,1$ & $83,4 \pm 3,3$ & $90,2 \pm 5,4$ & $94,4 \pm 5,0$ \\
\hline Grupo II & $58,4 \pm 2,8$ & $68,8 \pm 2,0$ & $77,6 \pm 1,4$ & $83,3 \pm 2,6$ & $81,6 \pm 4,2$ \\
\hline
\end{tabular}

Valores seguidos de * são estatisticamente diferentes dos valores em zero.

na desnervação acetabular, os ramos dos nervos obturador e femoral não seriam necessariamente afetados, e que a variação da localização deles já teriam sidos documentados em estudos anatômicos.

Um desses cães supracitados apresentava DAD acentuada bilateral, avaliada através do exame radiográfico no período pré-operatório. É de opinião dos autores que provavelmente a curetagem não tenha sido efetiva, já que havia uma alteração importante na articulação causada pela DAD, prejudicando a localização anatomica ideal, apesar da abordagem ter sido aberta. O outro cão passou por uma nova intervenção cirúrgica, ressecção da cabeça e colo femoral (RCCF), e obteve resultado satisfatório após 60 dias de pós-operatório, entretanto o proprietário referiu que o animal permaneceu com discreta alteração na deambulação.

Estudo recente avaliando de forma objetiva a ressecção da cabeça e colo femoral em cães demonstrou que ela não restabelece a função articular (OFF \& MATIS, 2010). No entanto, não se pode desconsiderar o efeito benéfico na qualidade de vida dos pacientes submetidos ao procedimento (PIERMATTEI, 2011).

Além da RCCF, outros procedimentos podem ser utilizados para cães com displasia coxofemoral, como a sinfiodese púbica, a osteotomia tripla, a osteotomia dupla da pelve, a osteotomia intertrocantérica e a implantação de prótese total, entre outras. Entretanto, tais procedimentos apresentam algumas limitações, como a idade do animal, o grau de atrofia muscular, a anatomia as estruturas envolvidas, os recursos financeiros do proprietário e habilidade do cirurgião (DUELAND et al., 2001). A literatura é categórica em afirmar que a técnica mais adequada para cães com displasia coxofemoral é a colocação de próteses articulares (KINZEL et al., 2002), porém, 
devido aos seus altos custos e necessidade de equipe especializada, ela é pouco difundida na realidade brasileira.

Em relação às complicações pós-cirúrgicas, três animais do GII tiveram deiscência da sutura, levando a um maior período de cicatrização, condição que pode ter interferido na avaliação das variáveis estudadas, visto que o local apresentava-se inflamado, entretanto, neste estudo, não foram relatadas maiores complicações associadas à deiscência. ROCHA (2005) refere um caso de luxação coxofemoral pós-cirúrgica e sugere que, após a cirurgia, há alívio da dor, de tal forma que o animal passa a usar o membro com firmeza. Essa situação, somada à atrofia muscular pré-existente, favoreceu a luxação coxofemoral. A restrição inicial de exercícios deve ser recomendada com rigor nos cães displásicos submetidos a DC para evitar a luxação, pois o grau de atrofia muscular e a distensão das estruturas de suporte podem favorecer essa ocorrência (MILLIS \& LEVINE, 1997). Ademais, a fibrose periarticular préexistente limita a excursão articular, e há de se ponderar se, nos casos de subluxação, a desnervação propicia os mesmos resultados.

Os autores sugerem que possa existir um viés no estudo, já que as escalas utilizadas são subjetivas e, dessa forma, podem deixar o estudo suscetível a efeitos placebo, bem como à influência dos pesquisadores na interpretação dos resultados. QUINN et al. (2007) compararam a acurácia de escalas de avaliação numérica e analógica visual com a análise de marcha por placa de força e concluíram que as escalas subjetivas de pontuação não substituem a avaliação por placa de força. Denotam ainda que cada observador utilize uma escala individual única e deva, portanto, permanecer o mesmo durante todo o estudo.

Recentemente, LISTER et al. (2009) avaliaram objetivamente as forças de reação do solo (FRS) em cães displásicos, bilateralmente, após a desnervação aberta unilateral, e demonstraram a eficácia da técnica em $50 \%$ dos cães, devido à melhora dos valores de FRS no membro operado e a uma diminuição significativa nos valores de FRS do membro controle, o que seria coerente ao compartilhamento da carga e aumento da perda compensatória pela claudicação.

LISTER et al. (2009) inferiram também que escalas subjetivas apresentaram baixa concordância entre os observadores quando comparadas ao FRS, apresentando interpretação similar somente em casos de claudicação grave. Cabe ressaltar que, apesar da força de carga no membro operado estar abaixo do esperado, as deformações articulares não são alteradas durante a desnervação, o que poderia explicar os achados dos autores.
Quanto à perimetria da coxa, obteve-se aumento numérico dos valores, que corresponde a uma melhora clínica na condição de massa muscular. A extensão também evoluiu positivamente, com aumento numérico dos valores, e flexão com valores diminuídos que significou maior capacidade de flexionar o membro. Por conseguinte, com a evolução dos valores de extensão e flexão, os cães obtiveram maior APM. Apesar dessas observações, não foram evidenciadas diferenças estatísticas nestas variáveis ao longo do período de avaliação do estudo.

De acordo com estudo de JAEGGER et al. (2002), a goniometria é método objetivo e confiável para a determinação da amplitude de movimento das articulações saudáveis e, quando comparada dentro de uma mesma raça, apresenta resultados ainda mais fidedignos. Denotam ainda que a sedação não pareceu influenciar na APM, porém sua amostra utilizava apenas animais saudáveis. Ao contrário do trabalho citado, no presente estudo, utilizou-se a goniometria em cães de diferentes raças e com alterações morfológicas e sensitivas na articulação, sugerindo, portanto, que os resultados podem sofrer algum tipo de interferência de acordo com essas variáveis.

A utilização da DC insere-se com grande vantagem devido à mínima invasão, curto período transoperatório e de convalescença, podendo ser utilizada em animais de qualquer idade, não necessitando de instrumentos cirúrgicos especiais para sua aplicação (KINZEL et al., 2002a). Uma desvantagem da técnica está no fato de que só se alteram os sinais clínicos e não a evolução ou cura da doença (KINZEL et al., 1998 e 2002b; SCHMAEDECKE, 2004).

Visto a similitude entre as abordagens, é possível escolher uma das técnicas de acordo com a individualidade de cada caso. A DC percutânea tem um menor tempo cirúrgico, menor incisão e, consequentemente, menor exposição à contaminação. Todavia, a habilidade do cirurgião e grau de alteração morfológica do acetábulo em decorrência da DAD devem ser levados em consideração.

\section{CONCLUSÃO}

Baseado nos achados deste estudo, conclui-se que a abordagem percutânea ou aberta da DC produz resultados similares nas variáveis avaliadas e ambas resultam em remissão significativa da sintomatologia associada à displasia coxofemoral.

\section{AGRADECIMENTOS}

A concessão de bolsa de iniciação científica através do Programa Institucional de Iniciação Científica e Conselho Nacional de Desenvolvimento Científico e Tecnológico (PIBICCNPq). 


\section{REFERÊNCIAS}

ARNBJERG, J. Recent information about hip dysplasia. Vet Clin North Am: Small Anim, v.29, n.4, p.921-934, 1999.

BRAUN, D. et al. Dorsale denervation der hüftgelenkskapsel beim hund ergebnisse einer langzeitstudie. Kleintierpraxis, v.4, p.185-244, 2003.

DUELAND, R.T. et al. Effects of pubic symphysiodesis in dysplastic puppies. Vet Surg, v.30, p.201-217, 2001.

FERRIGNO, C.R.A. et al. Estudo da técnica de denervação da cápsula articular coxofemoral no tratamento da dor em cães com displasia coxofemoral: resultados preliminares. Braz J Vet Res Anim Sci, v.41, supl., p.169-170, 2004.

FERRIGNO, C.R.A., et al. Denervação acetabular cranial e dorsal no tratamento da displasia coxofemoral em cães: 360 dias de evolução de 97 casos. Pesq Vet Bras, v.27, n.8, p.333340, 2007b. Disponível em: <http://www.scielo.br/ scielo.php? script=sci_art text \& pid=S 0100 736X2007000800003>. Acesso em: 30 ago. 2011. doi: 10.1590/S0100-736X2007000800002.

HUDSON, J.T. et al. Assessing repeatability and validity of a visual analogue scale questionnaire for use in assessing pain and lameness in dogs. Vet Res, v.65, p.1634-1643, 2004. Disponível em: <http://avmajournals.avma.org/doi/abs/10.2460/ ajvr.2004.65.1634>. Acesso em: 30 ago. 2011. doi: 10.2460/ ajvr.2004.65.1634.

HULSE, D.A.; JOHNSON, A.L. Tratamento da doença articular. In: FOSSUM, T.W. Cirurgia de pequenos animais. São Paulo: Roca, 2002. p.1042-1050.

JAEGGER, G. et al. Reliability of goniometry in labrador retrievers. Vet Res, v.63, p.979-986, 2002. Disponível em: <http://www.utc.edu/Faculty/David-Levine/Goniometry.pdf>. Acesso em: 30 ago. 2011.

KINZEL, S. et al. Sensory innervation of the hip joint capsule in dogs. Tierarz Praxis, v.26, p.330-335, 1998.

KINZEL, S. et al. 10 years experience with denervation of the hip joint capsule for treatment of canine hip joint dysplasia and arthrosis. Berl Munch Tierarzt Wochen, v.115, p.5356, 2002a.

KINZEL, S. et al. Clinical evaluation of denervation of the canine hip joint capsule: a retrospective study of 117 dogs. Vet Comp Orthop Traumatol, v.15, p.51-56, 2002b.

MILLIS, D.L.; LEVINE, D. The role of exercise and physical modalities in the treatment of osteoarthritis. Vet Clin North Am: Small Anim, v.27, p.4, p.913-930, 1997.

OFF, W.; MATIS, U. Excision arthroplasty of the hip joint in dogs and cats. Clinical, radiographic, and gait analysis findings from the Department of Surgery, Veterinary Faculty of the Ludwig-Maximilians-University of Munich, Germany. Vet Comp Orthop Traumatol, v.23, n.5, p.297-395, 2010. Disponível em: <http:/www.orthovetsupersite.org/system/files/ vcot_2010-23-5_13649_0.pdf>. Acesso em: 30 ago. 2011.

PIERMATTEI, D. Excision arthroplasty of the hip joint in dogs and cats. Vet Comp Orthop Traumatol, v.24, n.1, p.89, 2011.

QUINN, M.M. et al. Evaluation of agreement between numerical rating scales, visual analogue scoring scales, and force plate gait in dogs. Vet Surg, v.36, p.360-307, 2007. Disponível em: <http://msg.vu-wien.ac.at/uploads/media/Quinn.pdf>. Acesso em: 30 ago. 2011. doi: 10.1111/j.1532950X.2007.00276.x.

LISTER, S.A. et al. Ground reaction force analysis of unilateral coxofemoral denervation for treatment of canine hip dysplasia. Vet Comp Orthop Traumatol, v.22, p.137-141, 2009. Disponível em: <http://www.schattauer.de/de/magazine/ uebersicht/zeitschriften-a-z/vcot/contents/archive/issue/127/ manuscript/11089.html>. Acesso em: 30 ago. 2011. doi: 10.3415/VCOT-08-02-0024.

RAWSON, E.A. et al. Simultaneous bilateral femoral head and neck ostectomy for the treatment of canine hip dysplasia. $\mathbf{J}$ Am Animal Hosp Assoc, v.41, p.166-170, 2005.

ROCHA, L.B.; TUDURY, E.A.O. Denervação articular em cães com osteoartrose coxofemoral secundária a displasia. 2005. 85f. Dissertação (Mestrado em Ciência Veterinária) - Programa de Pós-graduação em Ciência Veterinária, Universidade Rural Federal do Pernambuco, Recife. Disponível em: <http://www.scielo.br/pdf/cr/v39n2/ a61cr184.pdf $>$. Acesso: 01 set. 2011.

SCHMAEDECKE, A. Estudo quantitativo das fibras nervosas do periósteo acetabular em cães. 2004. $103 \mathrm{f}$. Dissertação (Mestrado em Anatomia dos Animais Domésticos) - Programa de Pós-graduação em anatomia dos animais domésticos e silvestres, Universidade de São Paulo, SP. Disponível em: <http://www.teses.usp.br/teses/disponiveis/10/ 10132/tde-19102004-085949/pt-br.php>. Acesso em: 01 set. 2011.

SCHULZ, K.S. Displasia coxofemoral. In: FOSSUM, T.W. Cirurgia de pequenos animais. 3.ed. Rio de Janeiro: Mosby Elsevier, 2008. p.1233-1246.

SELMI, A.L. et al. Denervação capsular percutânea no tratamento da displasia coxofemoral canina. Ciênc Rural, v.39, p.460-466, 2009. Disponível em: <http://www.scielo.br/ pdf/cr/v39n2/a61cr184.pdf>. Acesso: 01 set. 2011.

TOMLINSON, J.; MCLAUGHLIN, R. Medically managing canine hip dysplasia. Vet Med, v.91, p.48-53, 1996. 